\title{
About traceability of the biochemical measurement results - some case studies on the metrological and practical aspects
}

\author{
Steluta Duta ${ }^{1, a}$ \\ ${ }^{1}$ National Institute of Metrology, 11 Vitan Barzesti, Sector 4, Bucharest, Romania
}

\begin{abstract}
The comparability of biochemical measurement results performed by any medical laboratories could be reached only when common reference systems can be established for worldwide use. There is recognised a critical step in the reference systems to achieve traceability of values obtained using reference measurement procedures and reference materials traceable to the universally recognized and accepted reference point such as SI units. The IVD Directive requires traceability of the values assigned to calibrators and/or control materials. It must be assured through available reference measurement procedure and/or available reference materials of a higher order. In this respect, the JCTLM promote and give guidance on internationally recognized and accepted equivalence of measurements in laboratory medicine and traceability to appropriate measurement standards. There are already established higher order metrological reference materials, reference measurement procedures as well as reference measurement services for some biochemical analytes. In laboratories medicine, the metrological traceability and the related metrological concept measurement uncertainty are not always possible to be established. In this paper some traceability case studies for biochemical constituents are presented in opposite with some case studies for hematology measurements performed in medical laboratories.
\end{abstract}

\section{Starting from some definitions}

As well known there are international activities at the high metrology level regarding the traceability in laboratories medicine. The main goal of obtaining comparability of medical laboratory results over time and space, either by the same measurement procedure or by different measurement procedures, could be reached only when common reference systems can be established for worldwide use. An important step in this process is to achieve the traceability of measured values using the reference measurement procedures and the reference materials to a universally recognized and accepted reference point such as the International System of Units (SI). As a consequence of these needs, the Joint Committee on Traceability in Laboratory Medicine (JCTLM) of International Bureau of Weights and Measures (BIPM) was created to promote and give guidance on internationally recognized and accepted equivalence of measurements in laboratory medicine and traceability to appropriate measurement standards [1].

Nowadays, the traceability issue for medical devices is endorsed by the European Directive 98/79/EC: In vitro diagnostic medical devices (IVD) [2] and by ISO 17511/2000: In vitro diagnostic medical devices -

\footnotetext{
${ }^{a}$ Corresponding author: xavier.mougeot@cea.fr
}

Measurement of quantities in biological samples Metrological traceability of values assigned to calibrators and control materials [3].

The directive states as the essential requirement that "The traceability of values assigned to calibrators and/or control materials must be assured through available reference measurement procedures and/or available reference materials of a higher order." (IVD 98/79/EC, Annex I, Part A-Clause 3).

The traceability is an essential requirement looking especially on the comparability of measurement data due to the impact of the non-traceable IVD measurement systems on the incorrect patient diagnosis and treatment, the impact that can not be easily estimated in the interpretation of clinical studies regarding the health care and patients health in such a way to not compromise the health and safety of patients, users and other third parties.

Currently, there are already established higher order metrological reference materials, reference measurement procedures as well as reference measurement services in the medicine laboratories. For practical reasons, it is important to notice from the above definition that the directive refers to the ,available" reference materials and reference measurement procedures when dealing with 
assignment of value to calibrators and control materials. It is due to the difficulties and challenges to put it in place, due to various factors, such as economical and technical factors.

The most recent certified reference materials traceable to the SI units such as electrolytes, drugs, metabolites and substrates, enzymes, proteins, etc. and reference measurement procedure for well-defined chemical entities or internationally recognised reference method-defined measurand are published on the BIPM website [1]. In laboratories practice they are shortly called primary reference materials (PRM). Apart of these information there are available also the reference materials with the assigned values based on an internationally agreed protocol, but not SI-traceable. Not internationally recognised reference measurement procedures exist, for instance for reference materials for blood typing, coagulation factors, microbial serology, nucleic acids etc.

For laboratories practical use, there are also various manufacturers (Bio-Rad, Bio-Systems, Roche, etc.) that provide kinds of calibrators and quality control materials for clinical chemistry, haematology and immunoassay control materials. It has long been recognised by manufactures and laboratory professionals that significant differences occur in the test results due to the measurement methods applied analytical working procedures as well as instrument's performances.

There are measurement procedures internationally recognised as reference for some biochemical constituents such as glucose, urea, cholesterol, creatinine, triglycerides, etc. The procedure is based on the isotope dilution- gas chromatography-coupled with mass spectrometry technique (ID-GC-MS). For some electrolytes such as calcium, iron, lead etc. the measurement procedures internationally recognised as reference procedure is the isotope dilution-inductive coupled plasma spectrometry-coupled with mass spectrometry (ID-ICP-MS) and (isotope dilution)thermal ionisation mass spectrometry (ID)-(TIMS), etc.

In this context, it is wise to mention the commutability concept of the reference materials, usually applied to calibrators and/or reference materials, and/or control materials, defined by [4] as ,, the property of a reference material, demonstrated by the closeness of agreement between the relation among the measurement results for a stated quantity in this material, obtained according to two given measurement procedure, and the relation obtained among the measurement results for other specified materials". This concept should be considered especially for the group of purified substances which, due to the absence of reference measurement procedures, should not be directly used for calibration of routine methods unless commutability is established and/or matrix effect independent internationally recognized standardized value transfer protocols to commutable samples are applied. The problem has been addressed to the laboratory works as well as in the external quality controls, when the need for creating peer-groups grading for such different laboratory methods appear.
In accordance with [4] the metrological traceability is „, the property of a measurement result whereby the result can be related to a reference through a documented unbroken chair of calibrations, each contributing to the measurement uncertainty”. The term ,calibrator” is only used in certain fields, with the meaning of ,the measurement standard used in calibration" Further, the ILAC document [5] on the traceability issue ,considers the elements for confirming metrological traceability to an unbroken metrological traceability chain to an international measurement standard or to a national measurement standard, a documented measurement uncertainty, a documented measurement procedure, accredited technical competence, metrological traceability to the SI and calibration intervals"

Appling these concepts to the calibrator traceability, this means that a calibrator's assigned value must by systematically derived from reference materials or reference methods in an unbroken chain of comparisons, based on the documented measurement procedure and the detailed measurement uncertainty evaluation.

\section{Practical aspects of metrological traceability in clinical chemistry}

The metrological traceability in laboratories medicine and the related metrological concepts described above are not always possible to be established in a metrological manner for all clinical chemistry analytes, haematology and/or immunology. As underlined above, the concept of metrological traceability, as firmly embedded in the IVD Directive, is an essential requirement.. Traceability of the assigned value of a calibrator must be systematically derived from higher order characterised material through an unbroken chain of reference measurement procedure and reference materials, each step in the chain having stated uncertainty.

From practical point of view there are important implications for the assignment of value to calibrators that are based on human serum and contain purified additives from human and animal origin, purified pharmaceuticals and chemical additives. The comparisons chain is often broken due to the material preparation, i.e. liofilization, freezen, etc. even the preparation steps are based on the detailed protocol for assigning the quantity value.

Even the mulicalibrator's manufacturers give the information regarding the source of traceability of some analytes, there are information missing for other many analytes. The metrological traceability source for 14 analytes contained in a lyophilized calibrator serum (Table 1), from a total of above 30 analytes usually contained in the multicalibrator, is documented. In this case, the results comparability of all analytes based on the the multicalibrator used can not be automatically assumed due to the lack of their demonstrated traceability. 
Table 1:The traceability sources in various manufacturer's multicalibrators

\begin{tabular}{|c|c|c|c|}
\hline Analyte & $\begin{array}{c}\text { Bio- } \\
\text { System }\end{array}$ & Roche & Dia-Sys \\
\hline Albumin & $\begin{array}{l}\text { SRM } \\
927 d\end{array}$ & CRM 470 & CRM 470 \\
\hline $\begin{array}{l}\text { Bilirubin, } \\
\text { Total }\end{array}$ & $\begin{array}{l}\text { SRM } \\
909 b\end{array}$ & SRM 916a & SRM 616a \\
\hline Calcium & $\begin{array}{l}\text { SRM } \\
909 \mathrm{~b}\end{array}$ & $\begin{array}{l}\text { AAS } \\
\text { method }\end{array}$ & $\begin{array}{c}\text { AAS } \\
\text { calibrated } \\
\text { with PRM }\end{array}$ \\
\hline Chloride & $\begin{array}{l}\text { SRM } \\
909 b\end{array}$ & $\begin{array}{c}\text { Coulometry } \\
\text { method }\end{array}$ & $\begin{array}{c}\text { Coulometry } \\
\text { calibrated } \\
\text { with PRM }\end{array}$ \\
\hline Cholesterol & $\begin{array}{l}\text { SRM } \\
909 b\end{array}$ & GC-IDMS & IDMS \\
\hline Creatinine & $\begin{array}{l}\text { SRM } \\
909 b\end{array}$ & GC-IDMS & IDMS \\
\hline Glucose & $\begin{array}{l}\text { SRM } \\
965 b\end{array}$ & IDMS & IDMS \\
\hline Magnezium & $\begin{array}{l}\text { SRM } \\
909 b\end{array}$ & $\begin{array}{l}\text { AAS } \\
\text { method }\end{array}$ & $\begin{array}{c}\text { AAS } \\
\text { calibrated } \\
\text { with PRM }\end{array}$ \\
\hline Potassium & $\begin{array}{l}\text { SRM } \\
909 \mathrm{~b}\end{array}$ & - & $\begin{array}{c}\text { FP } \\
\text { calibrated } \\
\text { with PRM } \\
\end{array}$ \\
\hline Sodium & $\begin{array}{l}\text { SRM } \\
909 b\end{array}$ & - & $\begin{array}{c}\text { FP } \\
\text { calibrated } \\
\text { with PRM }\end{array}$ \\
\hline Protein, Total & $\begin{array}{l}\text { SRM } \\
927 b\end{array}$ & $\begin{array}{l}\text { Biuret } \\
\text { method }\end{array}$ & - \\
\hline Triglycerides & $\begin{array}{l}\text { SRM } \\
909 b\end{array}$ & GC-IDMS & SRM 909b \\
\hline Urea & $\begin{array}{l}\text { SRM } \\
909 \mathrm{~b}\end{array}$ & SRM 909 b & SRM 909b \\
\hline Uric acid & $\begin{array}{l}\text { SRM } \\
909 b\end{array}$ & GC-IDMS & IDMS \\
\hline
\end{tabular}

Note: The information from this table is not from the most recent lots of calibrator

\section{Abbreviations:}

SRM-Standard Reference Materials, NIST-SUA

CRM-Certified Reference Materials, European Communities

PRM- Primary Reference Material

AAS-Atomic Absorption Spectrometry

FP- Flame Photometry

GC-Gas Chromatography

IDMS-Isotope Dilution Mass Spectrometry

It should be also mentioned that information regarding the uncertainty associated to the multicalibrators's assigned values are missing, the measurement uncertainty components are not fully identified and estimated, as a consequence, the traceability of the patients result to the calibrator is not demonstrated.

In the following paragraphs some practical aspects regarding the metrological as well as routine glucose measurements. The glucose measurements are commonly used in the diagnosis and treatment of diabetes. Glucose measurements are either done in the reference laboratories/institutes, in the clinical medical laboratories or by self-monitoring glucose meters and in hospital by continuous monitoring glucose meters.

In the reference laboratories as well as in few prestigious institutes, the metrological traceability for glucose measurement results is established at higher order metrological reference materials (SRM 917b, D-glucose or SRM 965a, Glucose in frozen human serum). The reference measurements [1] are based on Isotope Dilution-Gas Chromatography - Mass Spectrometry (ID-GC-MS) in the range of $(1 \ldots 20) \mathrm{mmol} / \mathrm{L}$ with the associated measurement uncertainty of $(0,5 \ldots 2,0) \%, \mathrm{k}=2$.

In biochemical laboratories the routine glucose measurements are usually performed against the calibration line of the photometric systems used. The calibration is performed by the instrument's manufacturer with the laboratory's multicalibrator. Calibration, is usually stable for approximately few days and is required with each change in the reagents lots. The calibration should be verified according with the established predefined acceptance criteria (for instance, slope, intercept and/or correlation coefficient) that are specific for each instrument.

The routine measurement procedure is based on the hexokinase/G-6-PDH reaction; the glucose (G) is phosphorylated by hexokinase (HK) in the presence of adenosine triphosphate (ATP) and magnezium ions to produce glucose-6-phosphate (G-6-P) and adenosine diphosphatare (ADP). Glucose-6-phosphate dehydrogenase (G-6-P-PDH) specifically oxidizes G-6-P to 6-phosphogluconate with the concurrent reduction of nicotinamide adenine dinucleotide (NAD) to nicotinamide adenine dinucleotide reduced (NADH). One $\mu$ mole of $\mathrm{NADH}$ is produced for each $\mu$ mole of glucose consumed. The NADH produced is detected photometric at $340 \mathrm{~nm}$ against the calibration line.

Glucose + ATP ---HK----> 6- glucose phosphate +ADP

6- glucose phosphate $+N A D^{+}$-----> 6-phosphategluconate $+\mathrm{NADH}+\mathrm{H}^{+}$

An interlaboratories comparisons was performed using a synthetic material produced by INM [6], with the assigned glucose value of $(89 \pm 6) \mathrm{mg} / \mathrm{dl}, \quad k=2$ characterised in accordance with [7], following the next steps:

- The identification and selection of the participating laboratories, which perform the measurements in the appropriate environmental conditions as well as which follow the general rules of good analytical practice;

- The selection of participating laboratories (p laboratories) that are able to demonstrate the tracing of the internal calibration of the measuring instruments, that implement the internal control of their measurements as well as that they use the analytical methods within the available rea- 
gent kits which are guaranteed by their equipment suppliers;

- $\quad$ Running the measurements by the participating laboratories in accordance within the accepted protocol (i.e. five repeated measurements $(n=$ 5 ) in each sample and each laboratory report the individual concentration values $\left(\mathrm{x}_{\mathrm{ij}}\right)$ the mean concentration reported by the laboratory $\left(\bar{x}_{i}\right)$ and its standard deviation $\left(s_{i}\right)$

- The preliminary evaluation of the results based on the comments received from individual laboratory, the results evaluation based on the technical reasons (i.e. average results biased with more than 10\%) and/or repeating the measurements, if acceptable.

- The final evaluation of results and the values assignment and their associated uncertainty based on the laboratories results by overall average $=$

values $\left(x_{i, j}\right)$ for each biochemical constituent and the overall standard deviation $\left(s_{i, j}\right)$.

For routine glucose measurements variability (repeatability and/or reproducibility) is presented in Figure 1.

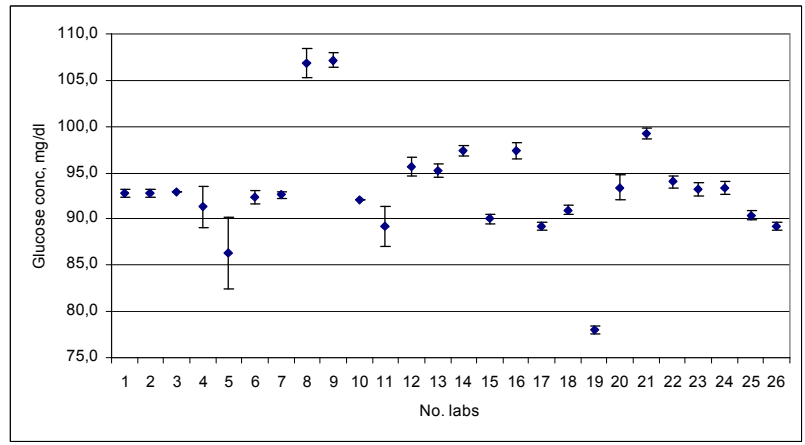

Fig.1: The routine glucose measurements on INM reference material Code 14.01

Various automatic clinical chemistry analysers were used in this exercise such as: Cobras 6000-C501 Cobras 6000 Roche, Cobras Integra 400 Plus (Roche), Hitachi 917 and 911, Hitachi 911 (Roche), Prestige 24i (Tokyo Boesky Medical), Beckman Synchrony CX9 Pro (Beckman), Chigwell (Awareness Technology), Architect ci4000; Architect ci8000 and Architect ci 8200 (Abbott), Konelab 20i (Thermo Clinical Laboratories), Dimension AR, Dimension RxLmax (Dade Behring) and others.

The overall mean value is about $93,2 \mathrm{mg} / \mathrm{dl}$ and the overall variability is about $12 \mathrm{mg} / \mathrm{dl}(\mathrm{k}=2)$

For self-monitoring glucose measurement or for the continuous monitoring glucose measurement, the certified reference materials in the form of lyophilized blood plasma are needed. The measurement procedures take place on the strip on solid deposited reagents. At the moment no certified glucose reference material of complex liquid biological composition exist and it is well know that the bias of these kind of measurements may be to $40 \%$ or even more from the reference value.

\section{Practical aspects on the traceability in haematology measurements}

As presented in the IVD Directive and discussed above for some biochemical constituents, 'the traceability of values assigned to calibrators ... must be assured through available reference measurement procedures and/or available reference materials of a higher order'

In haematology there are not defined primary measurement procedures, not primary reference materials for the components of the blood count. Usually, the haematology active ingredients are stabilised human red blood cells, human white blood cells and a platelet component in a preserved medium.

For haematology measurements, there are international conventional calibrators - whose values are not traceable to the SI but are assigned by international agreements, as well as international agreed reference measurement procedures, that are not SI traceable, but they are internationally agreed to be used as reference. The materials are international conventional calibrators based on fresh human blood [8].

The calibrator's hierarchy could be from the international conventional calibrator, to the manufacturer's working calibrator followed by the manufacturer's calibrator. The haematology calibrators usually can be used for WBC (white blood cells), RBC (red blood cells), PLT (platelets), HGB (haemoglobin) and HCT (hematocrit). But, apart of these particles, there are also present (NE) neutrophils, LY (lymphocytes), MO (monocytes), EO (eusinophils), BA (basophiles), PCT (playelecrit) that are not traceable values.

In the mean time, it is also important to mention the role and use of haematology control materials. The laboratories frequently use the haematology control materials for the regular checking of the instruments, whose values are assigned based on the manufacture's standing measurement procedure. For instance, the haematology results for Bio Rad control blood materials (low, normal and high level) using Sysmex KX-21 are presented in Table 2.

Table 2:The haematology results using Sysmex KX-21

\begin{tabular}{|c|c|c|c|}
\hline Analytes & $\begin{array}{l}\text { Haemato- } \\
\text { logy } 16 \mathrm{~T} \\
\text { control } \\
\text { material } \\
\text { (Bio-Rad) }\end{array}$ & $\begin{array}{l}\text { Reference } \\
\text { values } \\
( \pm 3 \mathrm{~s})\end{array}$ & $\begin{array}{c}\text { Average } \\
\text { measured } \\
\text { values }( \pm 3 \mathrm{~s})\end{array}$ \\
\hline $\begin{array}{c}\mathrm{WBC}^{*} 10^{3} / \mathrm{mm}^{3} \\
\mathrm{RBC}^{*} 10^{6} / \mathrm{mm}^{3} \\
\mathrm{PLT}^{*} 10^{3} / \mathrm{mm}^{3} \\
\text { HGB, g/dL } \\
\text { HCT, } \%\end{array}$ & Low level & $\begin{array}{c}2,2 \pm 0,3 \\
2,45 \pm 0,15 \\
45 \pm 9 \\
7,4 \pm 0,3 \\
20,2 \pm 1,5 \\
\end{array}$ & $\begin{array}{c}2,9 \pm 0,2 \\
2,95 \pm 0,27 \\
- \\
7,6 \pm 0,2 \\
26,2 \pm 2,8\end{array}$ \\
\hline $\begin{array}{c}\mathrm{WBC}^{*} 10^{3} / \mathrm{mm}^{3} \\
\mathrm{RBC}^{*} 10^{6} / \mathrm{mm}^{3} \\
\mathrm{PLT}^{*} 10^{3} / \mathrm{mm}^{3} \\
\text { HGB, g/dL } \\
\text { HCT, } \%\end{array}$ & $\begin{array}{c}\text { Normal } \\
\text { level }\end{array}$ & $\begin{array}{c}7,3 \pm 0,5 \\
4,18 \pm 0,15 \\
265 \pm 25 \\
13,6 \pm 0,4 \\
36,8 \pm 2,5\end{array}$ & $\begin{aligned} 8,4 & \pm 0,2 \\
4,87 & \pm 0,20 \\
- & \\
13,5 & \pm 0,2 \\
46,4 & \pm 1,2\end{aligned}$ \\
\hline $\begin{array}{c}\mathrm{WBC}^{*} 10^{3} / \mathrm{mm}^{3} \\
\mathrm{RBC}^{*} 10^{6} / \mathrm{mm}^{3} \\
\mathrm{PLT}^{*} 10^{3} / \mathrm{mm}^{3} \\
\text { HGB, g/dL } \\
\text { HCT, } \%\end{array}$ & $\begin{array}{l}\text { High } \\
\text { level }\end{array}$ & $\begin{array}{c}19,0 \pm 1,5 \\
4,82 \pm 0,20 \\
599 \pm 45 \\
16,4 \pm 0,5 \\
44,1 \pm 3,5\end{array}$ & $\begin{array}{c}20,2 \pm 0,9 \\
5,56 \pm 0,27 \\
- \\
16,5 \pm 0,3 \\
54,4 \pm 2,5\end{array}$ \\
\hline
\end{tabular}


It is difficult to say about the comparability of hematology results obtained with other analyser and/or with other hematology control material. It could be done only if the measurements are performed taking into account the traceability aspects presented above, respectively, the common use of international agreed calibrators as well as international agreed reference measurement procedures.

\section{Conclusions}

The traceability concept is still a challenge in the medical laboratories for many human tested constituents. For the biochemical results in the medicine laboratories there are well established routes for traceability assurance, respectively, well established higher order metrological reference materials, reference measurement procedures as well as reference measurement services. Even so, the self-monitoring glucose measurements and the continuous glucose measurements still need more attention how the traceability concept could be applied.

In haematology the reliable certified reference materials are needed as important tools for comparability of measurement results. The use and role of multicalibrators always used in all type of comparative medical measurements are the essential tools to ensure the traceability of results via their own traceable values within the associated measurement traceability.

\section{References}

1. www.bipm.org/jetlm/jetlm-db

2. European Directive 98/79/EC: In vitro diagnostic medical devices, (1998)

3. ISO 17511: In vitro diagnostic medical devicesMeasurement of quantities in biological samplesMetrological traceability of values assigned to calibrators and control materials, (2000)

4. International vocabulary of metrology-Basic and general concepts and associated terms (VIM), (2007)

5. ILAC P-10:2013: Traceability of Measurement results, (2013)

6. S. Duta, E. Ionita, M. Cretu, M. Georgescu, Lucrari de intretinere a etaloanelor de referinta in domeniul Materialelor de Referinta tip ser sintetic, BRML-INM study, (2012)

7. ISO 35: 1989: Certification of reference materialsGeneral and statistical principles, (1989)

8. International Council for Standardization in Haematology,ICSH Secretariat, Leuven, Belgium, (1991) 